\title{
A Spectrum Allocation Mechanism Based on HJ-DQPSO for Cognitive Radio Networks
}

\author{
Zhu Jiang \\ Chongqing Key Lab of \\ Mobile Communications \\ Technology, Chongqing \\ University of Posts and \\ Telecommunications, \\ Chongqing, China \\ zhujiang_cqupt@163.com
}

\author{
Xiong Jiahao \\ Chongqing Key Lab of \\ Mobile Communications \\ Technology, Chongqing \\ University of Posts and \\ Telecommunications, \\ Chongqing, China \\ 602165121@qq.com
}

\author{
Chen Hongcui \\ Chongqing Key Lab of \\ Mobile Communications \\ Technology, Chongqing \\ University of Posts and \\ Telecommunications, \\ Chongqing, China \\ 1271103552@qq.com
}

\author{
Han Chao \\ Chongqing Key Lab of \\ Mobile Communications \\ Technology, Chongqing \\ University of Posts and \\ Telecommunications, \\ Chongqing, China \\ hancao.cool@163.com
}

\begin{abstract}
In cognitive radio network model consisting of secondary users and primary users, in order to solve the difficult multi-objective spectrum allocation issue about maximizing network efficiency and users' fairness to access network, this paper proposes a new discrete multi-objective combinatorial optimization mechanismHJ-DQPSO based on Hooke Jeeves (HJ) and Quantum Particle Swarm Optimization (QPSO) algorithm. The mechanism adopts HJ algorithm to local search to prevent falling into the local optimum, and proposes a discrete QPSO algorithm to match the discrete spectrum assignment model. The mechanism has the advantages of approximating optimal solution, rapid convergence, less parameters, avoiding falling into local optimum. Compared with existing spectrum assignment algorithms, the simulation results show that according to different optimization objectives, the HJ-DQPSO optimization mechanism for multi-objective optimization can better approximate optimal solution and converge fast. We can obtain a reasonable spectrum allocation scheme in the case of satisfying multiple optimization objectives.
\end{abstract}

\section{Categories and Subject Descriptors}

C.2.1[Computer-Communication Networks]:Network Architecture and Design Wireless communication, Distributed networks.

\section{General Terms}

Algorithms

\section{Keywords}

Cognitive radio, Spectrum allocation, Quantum particle swarm, Multi-objective optimization.

\section{INTRODUCTION}

In Cognitive radio networks(CRN)[1-2], In order to meet the demand of normal communication, multiple communication indicators are required. As a key issue, multi-objective resource optimization needs to be solved. In CRN, spectrum allocation [3-4] is an issue for multi-objective optimization. The whole process is a multi-objective spectrum allocation process to maximize network efficiency and users' fairness to access. In recent years, with the domestic and foreign further researcher for this aspect, some spectrum allocation mechanisms have been gradually proposed. These mechanisms can be divided into three categories which are the game theory[5], the algorithms based on collaboration[6-7] and the thoughts based on new intelligent optimization algorithms [8-11]. The theory can enhance spectrum utilization and obtain a good allocation scheme. But there are also certain contradictions among the methods. In more complex situations, the model based on the game theory is difficult to achieve Nash equilibrium. The mutual cooperation of each node need be considered in the thoughts based on collaborative optimization. What's more, each node' strategy will affect other neighboring users. Therefore, the process is complex and not stable. There exist some contradictions among the search field, fast convergence, and convergence precision in the new intelligent algorithms, so it is hard to achieve an overall optimal effect and is hard to approximate the global optimal solution during the limited time. Therefore, the further improvement is required in this regard.

In view of the above insufficient spectrum allocation methods, this paper proposes a spectrum allocation mechanism based on HJ-DQPSO optimization. This mechanism can realize the alternate between the global search and local search, prevent falling into the local optimum, and can approximate the optimal solution. Besides it has the advantage of less parameter and fast convergence. The simulation results show that the algorithm can approximate optimal solution, and effectively solves the disadvantage of the old spectrum allocation methods. So it can solve the problem of difficult spectrum allocation effectively.

\section{SYSTEM MODEL}

In our model, each secondary user (SU) senses primary users (PU) respectively, and obtains available spectrum and interference constraint according to the users' location. CRN collects available spectrum and interference constraint via the control channel or a base station. The SU can select available spectrum and also adjust transmit power to avoid interfering with neighboring primary users. Figure 1 illustrates a deployment consisted of 3 primary users and 9 secondary users where each PU occupy a channel. Each SU obtains idle spectrum to achieve dynamic spectrum access. Each PU and SU has an interference range with radius. In PU interference range, the channel can't be occupied by SU to avoid interfering with the PU. When the interference ranges of two adjacent SU overlap, they will cause interference with each other if they use the same channel. Therefore, the SU is controlled both the PU constraint and the adjacent SU constraint on the basis of the normal communication. In CRN, as PU's and SU's location may change, the spectrum availability will change accordingly. So 
we assume that environmental conditions such as users' location and available spectrum are static during the short period it takes to perform spectrum allocation in our model.

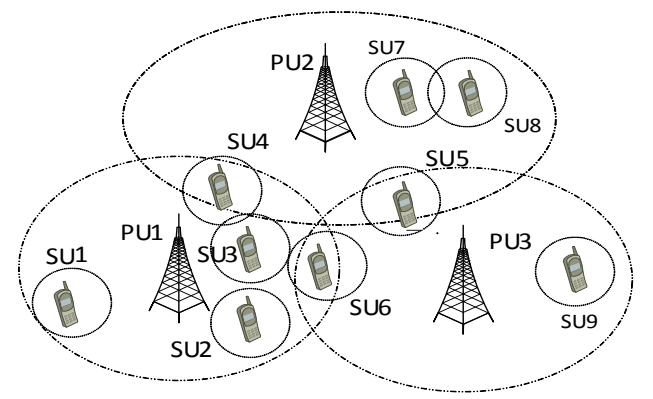

Figure 1 cognitive radio spectrum allocation model

\section{PROBLEM FORMULATION}

\subsection{Spectrum Allocation Model}

We assume a network of $\mathrm{N}$ secondary users and $\mathrm{M}$ primary users. Each SU and PU have an interference area with radius $d_{s}(\mathcal{N})$ which is bounded by the minimum and maximum transmit power, i.e. $\left[d_{\min }, d_{\max }\right]$ and $d_{p}(\mathcal{M}) . d_{p}(\mathcal{N}, \mathcal{M})$ represents the Euclidean distance from SU to PU, and $d_{s}\left(\mathcal{N}_{1}, \mathcal{N}_{2}\right)$ represents the Euclidean distance between $\mathrm{SU} \mathcal{N}_{1}$ and $\mathrm{SU} \mathcal{N}_{2}$. We define the key components of our model as follows:

Spectrum availability: $L=\left\{l_{n, m} \mid l_{n, m} \in\{0,1\}\right\}_{N \times M}$ represents the channel availability. $l_{n, m}=1$ if and only if channel $\mathrm{m}$ is available at user n. If $d_{p}(n, m)<d_{s}(n)+d_{p}(m)$, then $l_{n, m}=0$, otherwise $l_{n, m}=1$. Spectrum utility: $B=\left\{b_{n, m}\right\}_{N \times M}$ represents the channel utility. $b_{n, m}$ represents the utility that the user $n$ obtains from the spectrum $m$. The value of the spectrum efficiency can be obtained from the Euclidean distance between SU and PU, namely $b_{n, m}=d_{p}(n, m)^{2}$. Obviously, if $l_{n, m}=0$, then $b_{n, m}=0$.

Interference constraint: $C=\left\{\mathrm{c}_{n, k, m} \mid \mathrm{c}_{n, k, m} \in\{0,1\}\right\}_{N \times N \times M}$ represents the interference constraint among secondary users. $\mathrm{c}_{n, k, m}=1$ represents there will be some interference if the SU $n$ and the SU $k$ use the same spectrum simultaneously. There are certain constraints between interference constraint and spectrum availability, for example $c_{n, k, m} \leq l_{n, m} \times l_{k, m}$ and $c_{n, n, m}=1-l_{n, m}$. The binary model is used to represent the interference values in this paper. We assume that there are two secondary users $n$ and $k$ in this network, where their interference radius are $d_{s}(n)$ and $d_{s}(k)$ respectively, and the distance is $d_{s}(n, k)$ between the users. If $d_{s}(n, k)<d_{s}(n)+d_{s}(k)$, then $\mathrm{c}_{n, k, m}=1$. It represents that it will generate interference to use the same spectrum simultaneously. Otherwise, $\mathrm{c}_{n, k, m}=0$ means that it will not generate interference.

Allocation matrix without interference: $A=\left\{a_{n, m} \mid a_{n, m} \in\{0,1\}\right\}_{N \times M}$ represents the spectrum allocation, where $a_{n, m} \leq l_{n, m} \cdot a_{n, m}=1$ represents the spectrum $m$ is allocated to the SU $n$. The allocation matrix without interference needs to satisfy all the interference constraint defined by $\mathrm{C}$, namely if $c_{n, k, m}=1$, then $a_{n, m}+a_{k, m} \leq 1, \forall n, k<N, m<M$.

\subsection{System Optimization Objective}

This paper considers obtaining a reasonable spectrum allocation scheme in terms of the entire network. So this paper not only considers maximizing network utility, but also considering the fairness to access and the max proportional fairness. Therefore, the optimization objectives are defined as follows:

1) The Network Sum Utility $U_{N S U}$ is defined as:

$$
U_{N S U}=\sum_{n=1}^{N} \beta_{n}=\sum_{n=1}^{N} \sum_{m=1}^{M} a_{n, m} b_{n, m}
$$

2) Access Fairness $U_{A F}$ is defined as:

$$
U_{A F}=\sqrt{\frac{1}{N-1} \sum_{n=1}^{N}\left(\sum_{m=1}^{M} a_{n, m} b_{n, m}-\frac{1}{N} U_{N S U}\right)^{2}}
$$

3) Max-Proportional-Fairness $U_{M P F}$ is expressed as:

$$
U_{M P F}=\left(\prod_{n=1}^{N}\left(\sum_{m=1}^{M} a_{n, m} b_{n, m}+10^{-4}\right)\right)^{1 / N}
$$

4) Overall system performance $E$ which is weighted as the overall performance optimization is formulated as:

$$
E=w_{1} U_{N S U}-w_{2} U_{A F}+w_{3} U_{M P F}
$$

The spectrum allocation is to maximize network utilization $\mathrm{E}$ and to get the allocation matrix without interference $A^{*}$. Given the model above, we can define the spectrum allocation problem by the optimization function: $A^{*}=\arg \max E(A)$.Where $\varphi(L, C)_{N \times M}$ represents the spectrum allocation matrix set.

\section{SPECTRUM ALLOCATION BASED ON HJ-DQPSO OPTIMIZATION}

\subsection{Algorithm Analysis}

(1) The steps of HJ algorithm as follows:

Step1: Initializing the initial point $x^{0}$, step length $\delta^{0}=\left(\partial_{1}{ }^{0}, \partial_{2}{ }^{0}, \cdots, \partial_{n}{ }^{0}\right)^{T}$, acceleration coefficient $\gamma$, contraction coefficient $\theta \in(0,1)$, accuracy $\varepsilon$ and $\mathrm{k}=0$;

Step2: Starting from $y=x^{(k)}$, probing search successively according to the unit vector $e^{(j)}(j=1, \cdots, n)$. Then we choose the positive probing or negative probing according to the comparison of iteration value.

Step3: Ordering $y=x^{(k+1)}$, if $f\left(x^{(k+1)}\right)<f\left(x^{(k)}\right)$, then we choose pattern search for $x^{(k+1)}$ along with the acceleration direction that is $p^{(k)}=x^{(k+1)}-x^{(k)}$. Order $y=x^{(k+1)}+\gamma p^{(k)}$, return to step2, otherwise return to step4;

Step4: If $\left|\delta^{(k)}\right|<\varepsilon$, then the iteration is stopped and we export $x^{(k)}$. Otherwise we assume $y=x^{(k+1)}, x^{(k+1)}=x^{(k)}$, $\delta^{(k+1)}=\theta \delta^{(k)}$ and $k=k+1$, and return to step2.

(2) QPSO algorithm is an effective global optimization algorithm, which has a strong capability of optimization and faster convergence. The basic thought of QPSO is randomly to initialize a population whose size is $H$ in the $D$-dimensional space. Each particle which can constantly learn and save optimal value is 
regarded as a feasible solution. The position of each particle is $x_{i}^{t}=\left(x_{i, 1}^{t}, x_{i, 2}^{t}, \cdots, x_{i, D}^{t}\right)^{T}$, where the best position pbest of $x_{i, d}^{t} \in\left[L_{d}, U_{d}\right]$ is $p_{i}^{t+1}=\left(p_{i, 1}^{t}, p_{i, 2}^{t}, \cdots, p_{i, D}^{t}\right)^{T}$, the global best position gbest is $G_{g}^{t+1}=\left(p_{g, 1}^{t}, p_{g, 2}^{t}, \cdots, p_{g, D}^{t}\right)$. The iterative equation in the QPSO algorithm is expressed as:

$$
\begin{array}{r}
\text { mbest }=\frac{1}{H} \sum_{i=1}^{H} P_{i}(t)=\left(\frac{1}{H} \sum_{i=1}^{H} P_{i, 1}(t), \frac{1}{H} \sum_{i=1}^{H} P_{i, 2}(t), \cdots, \frac{1}{H} \sum_{i=1}^{H} P_{i, D}(t)\right) \\
P=\frac{\varphi_{1} p_{i}+\varphi_{2} G_{g}}{\varphi_{1}+\varphi_{2}} \\
X_{i, j}(t+1)=P(t) \pm \alpha \mid \text { mbest }_{j}(t)-X_{i, j}(t) \mid \ln \left(1 / u_{i, j}(t)\right)
\end{array}
$$

Where $\alpha$ is the factor of contraction and expansion and $u_{i, j}(t)$ is a random number from 0 to 1 ;

The QPSO algorithm described above is only suitable for continuous state model. But the spectrum allocation model in this paper is a discrete model, the QPSO algorithm is improved by discretization. We use the probability of 0 and 1 to improve the average optimal location mbest based on the equation (5). If the occurrence probability of 0 is large, then the value of mbest is 0 in the corresponding dimension, otherwise the value is 1 . If their probabilities are equal, then the value is a random value between 0 and 1.Besides we use equation (8) to replace the equation (6).

$$
\begin{gathered}
P_{i}=\varphi \text { pbest }+(1-\varphi) \text { gbest } \\
b=\beta \mid \text { mbest }-x_{i} \mid \ln (1 / u) \\
P_{r}=\left\{\begin{array}{l}
b / i \\
1, i f(b / i>1)
\end{array}\right.
\end{gathered}
$$

Where $\varphi$ is a random value between 0 and 1 and $u \sim U(0,1)$. We process the equation (7) by the crossover and mutation operation in the genetic algorithm to obtain equation (9) and (10). In the case of $P_{r}$ bigger than a $\operatorname{rand}()$, the corresponding dimension of $P_{i}$ use the cross conversion between 0 and 1, otherwise the $P_{i}$ isn't changed. Then we assign $P_{i}$ to the position of the particle $x_{i}$, process it with continuous iteration to find the optimal solution eventually.

(3) In order to solve the local optimum and improve search efficiency, the standard deviation $\mathrm{S}$ is introduced to determine whether the particles were trapped in local optimization status in this paper. The equation is defined as (11). Where $f_{i}$ is the fitness of particle $i, f_{\text {ave }}$ is equal to $\frac{1}{H} \sum_{i=1}^{H} f_{i}$.

$$
S=\sqrt{\frac{1}{H} \sum_{i=1}^{H}\left(f_{i}-f_{\text {ave }}\right)^{2}}
$$

\subsection{The procedure of HJ-DQPSO algorithm}

Step1: Initializing parameters and According to the improved equation (5) to obtain the discrete values, we can calculate the average best position of the population mbest;

Step2: According to equation (1), (2), (3), (4), we can calculate the fitness value of each particle;
Step3: Comparing the fitness of particle's individual best position pbest and global best position gbest ;

Step4: According to equation (8), (9), (10), we update the position of each particle;

Step5: According to equation (11), we calculate the standard deviation $\mathrm{S}$ of fitness of the population. If $S<\delta$, we will use the $\mathrm{HJ}$ algorithm to search. Then we will iterate the search value back into the DQPSO algorithm to continue searching;

Step6: If the stopping condition is reached, then the search will be finished. We will export the global best position and the global best fitness. Otherwise we return to the step 3 and continue to searching.

\section{SIMULATION ANALYSIS}

We randomly place primary and secondary users in a given area $(20 \times 20)$ which has $N$ SUs and $M$ PUs. In order to demonstrate the performance of the proposed algorithm, the proposed algorithm is compared with other algorithms which are the quantum genetic algorithm (QGA) proposed in paper [12], the particle swarm optimization (PSO) proposed in paper [11], the genetic algorithm (GA) proposed in paper [10] and the exhaustive search algorithm (ES). The simulation parameters refer to the corresponding literatures.

Figure 2 shows a cognitive wireless network model composed of 8 primary users and 15 secondary users simulation effect, the radius of $\mathrm{PU}$ and $\mathrm{SU}$ interference range is $d_{p} \in[3,5]$ and $d_{s} \in[1,3]$ respectively. The figure depicts the interference range distribution maps of the user. The spectrum availability, interference information and spectrum utility of the user can be presented from the simulation.

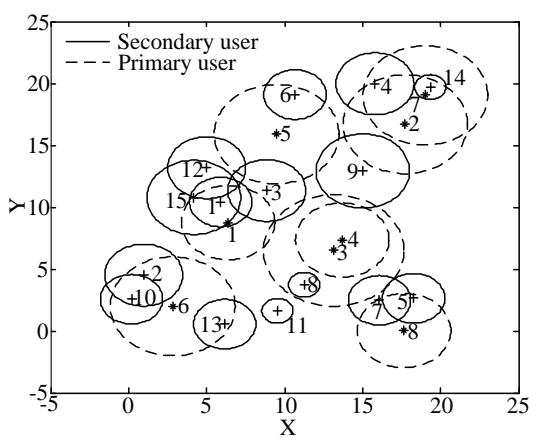

Figure 2 The distribution of interference range

The number of PU is set as 10 and the number of SU is set as 10 to 30 in the figure 3 . Under the overall efficiency of $U_{N S U}$ considered, we obtain average $U_{N S U}$ value of the 100 experiments. As can be seen from the results, the average value of the network sum reward showed an upward trend. The HJ-DQPSO algorithm is always higher than other algorithms and better approximates the optimal value. So it shows that the proposed spectrum allocation mechanism can be used to obtain a better spectrum allocation scheme.

The number of PU is set as 10 and the number of SU is set as 10 to 30 in the figure 4 . Under the overall efficiency of $U_{M P F}$ considered, we obtain average $U_{M P F}$ value of the 100 times experiments. As can be seen from the results, the average value of $U_{M P F}$ showed a downward trend in the case of a fixed number of 
spectrums. It suggests that the more secondary users, the more likely play a certain influence on the access fairness of the user. And the HJ-DQPSO algorithm proposed is better than other algorithms. This shows that this algorithm is superior to other algorithms in terms of max proportional fairness.

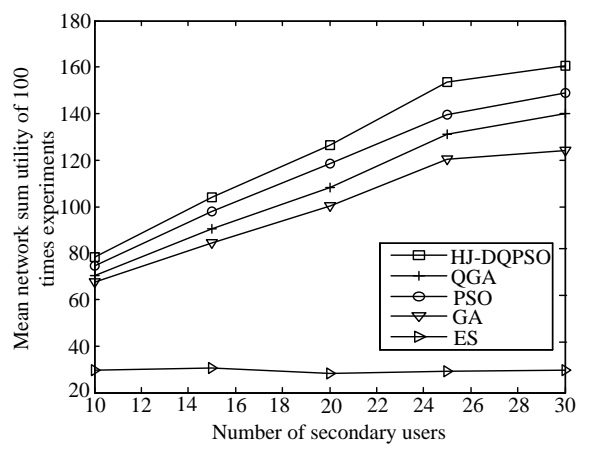

Figure 3 Mean $U_{N S U}$ with secondary users

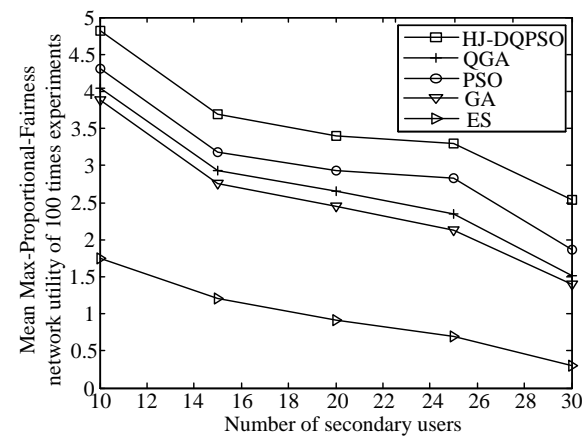

Figure 4 Mean $U_{M P F}$ with secondary users

Figure 5 shows that this scenario includes 10 primary users and 18 secondary users. The number of iterations is 200. The performance shows that the proposed algorithm can achieve a better performance than other algorithms by only considering the overall performance optimization. After a number of iterations, the algorithm seems to reach a final convergence. It shows that this algorithm has convergence. Therefore HJ-DQPSO algorithm can produce a better effect than other algorithms.

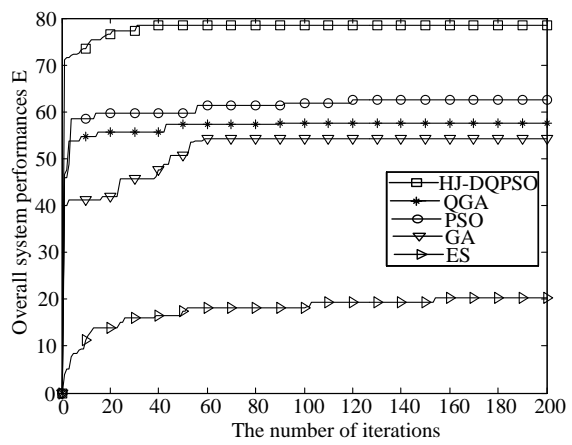

Figure 5 Overall system performances with iterations

\section{CONCLUSION}

In the complex heterogeneous networks, spectrum allocation is a key technique for the low spectrum utilization and the shortage of radio resource. This paper proposes a novel spectrum allocation mechanism based on HJ-DQPSO. Simulation results show that the proposed algorithm is an optimization method with strong optimization, fast convergence. Besides it is not easy to fall into local search and has certain stability. The proposed algorithm can provides a better spectrum allocation scheme with the overall system efficiency.

\section{ACKNOWLEDGMENTS}

This work is supported by The National Natural Science Foundation of China (61102062), The Key Project of Chinese Ministry of Education (212145), the Natural Science Foundation of Chongqing Science and Technology Commission (No.CSTC2011jjA1192), the Science and Technology Research Project of Chongqing Education Commission (No.KJ120530).

\section{REFERENCES}

[1] YC Liang, KC Chen, GY Li, and P Mhen, "Cognitive Radio: Networking and Communications: An overview", IEEE Transactions on Vehicular Technology, vol.60, pp. 33863407, 2011.

[2] LR Faganello, R Kunst, CB Both, LZ Granville, J Rochol. "Improving reinforcement learning algorithms for dynamic spectrum allocation in cognitive sensor networks", Wireless Communications and Networking Conference (WCNC), 2013 IEEE, On page(s): 35 - 40.

[3] H Zhu, AL Nel, M Sumbwanyambe, L Cheng. Revenue and utility maximization under centralized dynamic spectrum allocation. Industrial Engineering and Engineering Management (IEEM), 2013 IEEE International Conference on.

[4] Peng C, Zheng $\mathrm{H}$, Zhao B Y. Utilization and fairness in spectrum assignment for opportunistic spectrum access[J]. Mobile Networks and Applications, 2006, 11(4): 555-576.

[5] Sengupta S, Chatterjee M. Designing auction mechanisms for dynamic spectrum access [J]. Mobile Networks and Applications, 2008, 13(5): 498-515.

[6] Di Felice, M. ; Chowdhury, K.R. ; Bononi, L. Learning with the Bandit: A Cooperative Spectrum Selection Scheme for Cognitive Radio Networks Global Telecommunications Conference (GLOBECOM 2011), 2011 IEEE Publication Year: 2011, Page(s): 1-6

[7] Mengyi Liu, Huyin Zhang, Rongchao Fan, Zhenchen Duan. The GA Solution of Dynamic Spectrum Allocation in Cognitive Radio Based on Collaboration and Fairness. Circuits, Communications and System (PACCS), 2011 Third Pacific-Asia Conference on. 2011, Page(s): 1-4

[8] ZHANG B, ZHU Y, HU K. Spectrum assignment algorithm based on particle swarm optimization for cognitive radio[J]. Journal of Computer Applications, 2012, 31(12): 3184-3186.

[9] Zhao Z, Peng Z, Zheng S L, et al. Cognitive radio spectrum allocation using evolutionary algorithms[J]. IEEE Transactions on Wireless Communications, 2009, 8(9): 4421-4425.

[10] Gao Hong-Yuan, Li Chen-Wan. Membrane-inspired quantum bee colony algorithm for multi-objective spectrum allocation. [2014 Acta Physica Sinica 122802].

[11] CHAI Zheng-yi, LIU Fang. Spectrum allocation of cognitive wireless network based on immune clone. Journal on Communications. 2010(11). 